\title{
Properties of Geodesic Acoustic Modes and the Relation to Density Fluctuations
}

\author{
A. Krämer-Flecken, S. Soldatov, H. R. Koslowski, O. Zimmermann, and TEXTOR Team \\ Institut für Plasmaphysik, Forschungszentrum Jülich GmbH, EURATOM Association, D-52425 Jülich, Germany
}

(Received 27 January 2006; published 28 July 2006)

\begin{abstract}
The geodesic acoustic mode (GAM) is a high frequency branch of zonal flows, which is observed in toroidal plasmas. Because of toroidal curvature effects, density fluctuations are excited, which are investigated with the $O$-mode correlation reflectometer at TEXTOR. This Letter reports on the poloidal distribution of GAM induced density fluctuation and compares them with theoretical predictions. The influence of the GAM flows on the ambient turbulence is studied, too.
\end{abstract}

DOI: 10.1103/PhysRevLett.97.045006

PACS numbers: 52.35.Ra, 52.25.Gj, 52.35.Fp, 52.70.Gw

Zonal flows (ZFs) are self-generated poloidally and toroidally extended time varying sheared $E \times B$ flows in tokamak plasmas, which are radially localized. They arise from fluctuations of the electrostatic potential. From theory [1] two major branches of zonal flows are expected: the zero frequency zonal flow and the high frequency geodesic acoustic mode (GAM) [1,2]. In terms of the poloidal and toroidal mode number the GAM is supposed to have a $(m=1, n=0)$ structure in the electron density because pressure asymmetries on a flux surface are induced by nonuniform $E \times B$ flows. ZFs have gained increasing interest in recent years because they interact with the ambient turbulence. The current understanding assumes that the increased $E \times B$ shear by the ZFs overcomes the decorrelation rate of the ambient turbulence and influences the generation of transport barriers.

The GAM induced density $\left(\tilde{n}_{\mathrm{GAM}}\right)$ and the radial electric field $\left(\tilde{E}_{r}\right)$ fluctuations are directly measured with heavy ion beam probes $[3,4]$. At the plasma periphery the potential and density fluctuations are measured with Langmuir probes [5,6]. Beam emission spectroscopy [7] and correlation reflectometry (CR) [6] yield information on the density fluctuations and their propagation. Doppler reflectometry [8] measures $\tilde{E}_{r}$ connected with the perpendicular velocity oscillation $\left(\tilde{v}_{\mathrm{GAM}}\right)$. ZFs are detected mainly at the plasma edge [8] but also in the confinement region [9]. At the plasma edge the GAM induced relative density fluctuations are of the order $0.25 \%$ [5].

The spatial structure of the GAM is still not fully investigated. This Letter reports on the poloidal distribution of the GAM induced density fluctuations and compares them with theoretical predictions. Furthermore, the influence of the GAMs on the ambient turbulence is studied. We demonstrate that the single lines in the reflectometer spectra fulfill the ion-sound-speed scaling for GAMs and the radial localization as well.

TEXTOR (major radius $R_{0}=1.75 \mathrm{~m}$ and small radius $a=0.47 \mathrm{~m}$ ) is a tokamak with a toroidal belt limiter. It is equipped with an $O$-mode CR system [10] for turbulence studies, which can measure simultaneously in the equatorial plane $\left(\theta=0^{\circ}\right)$ and on the top of the vacuum vessel $\left(\theta=90^{\circ}\right)$. Each array consist of five antennas focused to the center of the vessel (Fig. 1). The frequency of the probing wave varies in $26-37 \mathrm{GHz}$; it corresponds to $0.86 \leq n_{c} \leq 1.7 \times 10^{19} \mathrm{~m}^{-3}$, where $n_{c}$ is the local density at the cutoff position. For data analysis spectral, correlation and statistical methods are applied. The turbulence propagation is calculated from the delay $\Delta t$ between the signals of two antennas spaced poloidally by $\Delta \theta$ according to $\Omega=$ $\Delta \theta / \Delta t$. The parameters of the ohmic plasmas are plasma current $300 \leq I_{p} \leq 400 \mathrm{kA}$, toroidal magnetic field $1.9 \leq$ $B_{T} \leq 2.25 \mathrm{~T}$, and line averaged density $1.5 \times 10^{19} \leq$ $\bar{n}_{e} \leq 2.8 \times 10^{19} \mathrm{~m}^{-3}$.

Figure 2 shows details of the amplitude, cross phase $(\phi)$, and coherence spectrum. A single line at $f \approx 17 \mathrm{kHz}$ with full width at half maximum (FWHM) of $\approx 3 \mathrm{kHz}$ in Figs. 2(a) and 2(c) implies a coherent oscillations in the density. The data are obtained from two antennas of the top array, spaced by $\Delta \theta=0.1 \mathrm{rad}$. The amplitude of the GAM is defined as the height of the GAM peak above the

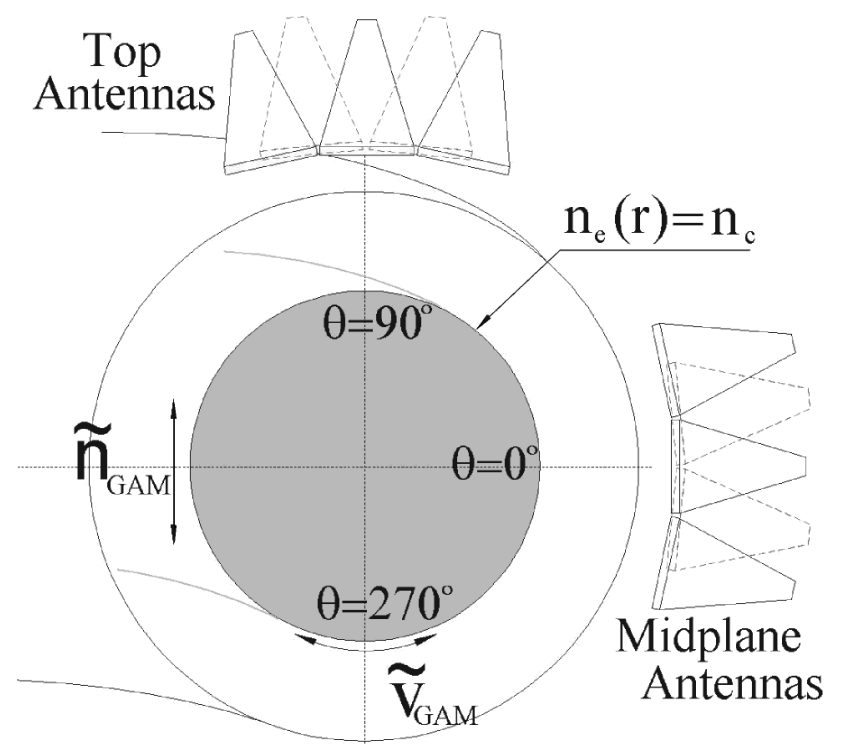

FIG. 1. Poloidal cross section with reflectometer antennas and a sketch of $\tilde{v}_{\mathrm{GAM}}$ and $\tilde{n}_{\mathrm{GAM}}$ fluctuations. 


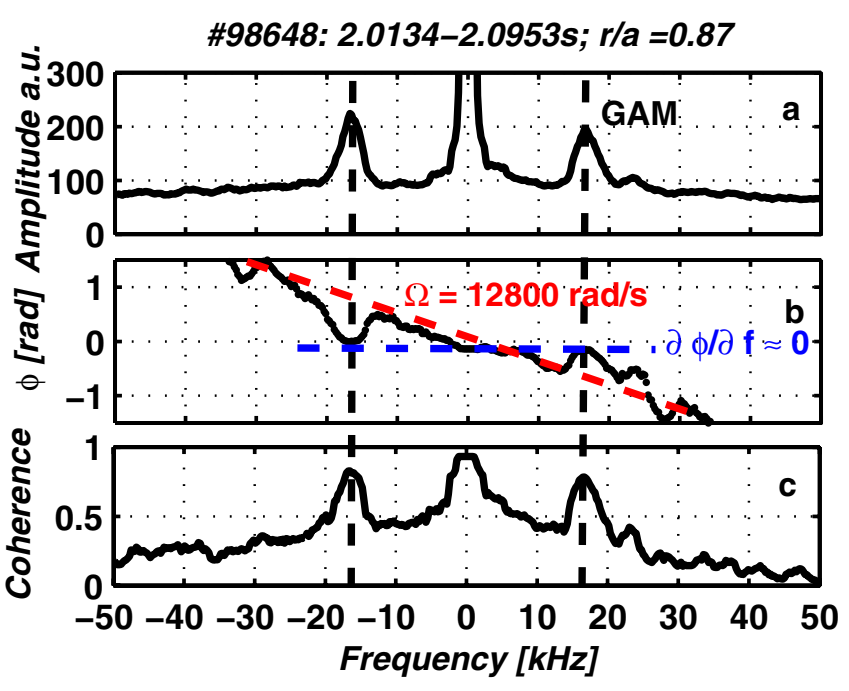

FIG. 2 (color online). Spectra of amplitude (a), cross phase (b), and coherence (c). In (a) and (c) a peak at $16.7 \mathrm{kHz}$ is seen. In (b) the change of the slope by the GAM is shown.

background turbulence in Fig. 2(a). The peak centered at $f=0$ results from magnetohydrodynamic (MHD) modes and plasma column movements. The zero frequency ZF [11] may also contribute. However, it is not a subject of the present Letter. The angular velocity of the turbulence is obtained from the cross-phase spectrum, where the slope is proportional to the poloidal time delay. At the GAM frequency we found $\partial \phi / \partial f \approx 0$, which corresponds to $\Delta t \approx$ 0 . Thus, the GAM density oscillations have no (or very small) phase shift, which implies a low $m$-number structure. No evidence for magnetic fluctuation accompanying the GAM induced density fluctuations is found by a Mirnov coil, positioned close to the antennas array at $\theta=$ $90^{\circ}$. Taking into account the efficiency of the Mirnov coil and the distance between the coil and the position of the GAM, the magnetic field perturbation is estimated to be $\tilde{B} / B \leq 3 \%$.

The frequency of the GAM changes with the inward moving reflection layer. This implies a scaling with the local plasma parameters. Our data follow the scaling of the GAM frequency with the ion-sound speed: $f_{\mathrm{GAM}}=\frac{k}{2 \pi R_{0}} c_{s}$ with $c_{s}=\sqrt{\left(T_{e}+T_{i}\right) / M_{i}}$, where $k$ is a constant in the range $1 \leq k \leq \sqrt{2}$ depending on the plasma elongation [8] and $T_{e}$ and $T_{i}$ are electron and ion temperature, respectively. The different lines in Fig. 3 represent the scalings for different ion mass. The dashed line is for pure deuterium, the solid line for hydrogen, and the dash-dotted line represents helium plasmas. The majority of the plasmas are found between the hydrogen and deuterium scalings and results from different $H / D$ ratios. The radial localization is analyzed from the radial development of the GAM amplitude. The GAM is visible in the range $0.80 \leq r / a \leq 0.91$. The position with the highest amplitude is found for $r / a=$ $0.88 \pm 0.01$. This is in agreement with measurements from

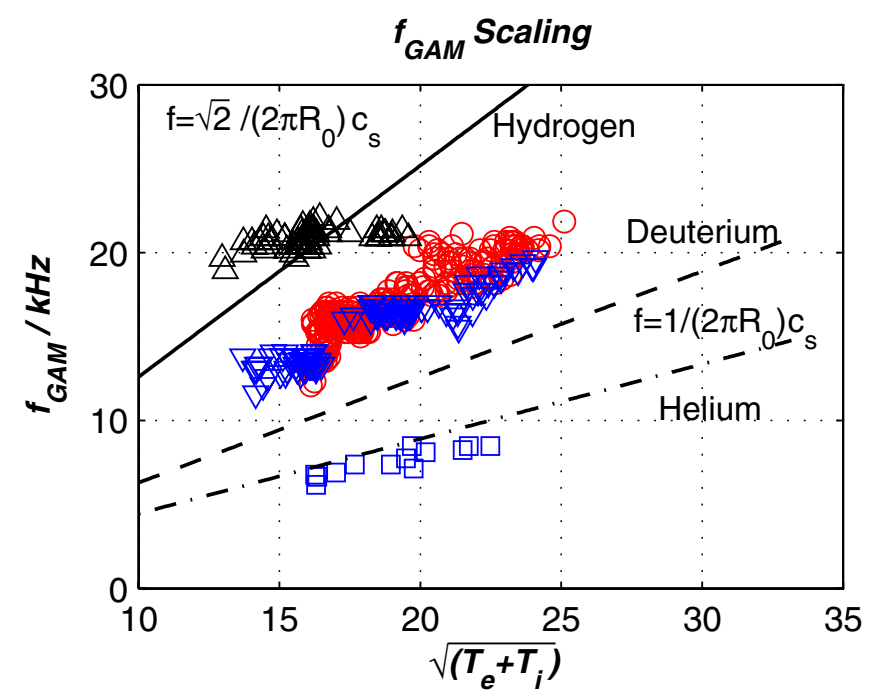

FIG. 3 (color online). Scaling of $f_{\mathrm{GAM}}$ with $\sqrt{T_{e}+T_{i}}$. The fits represent different ion mass. $(\square)$ denotes helium and $(\triangle)$ hydrogen plasmas. Other symbols denote deuterium plasmas with different $\mathrm{H} / \mathrm{D}$ ratios.

DIII-D [5]. Towards the plasma edge the amplitude of the GAM decreases fast, within $0.01 \mathrm{~m}$. At a reflection layer $r_{c} / a>0.91$, no indication of GAMs is found.

We note here that the observed frequency scaling and the radial localization meet the conditions for GAMs as reported in $[5,8,9,12]$.

For the GAM induced density fluctuations theoretical models [1] predict a $\sin (\theta)$ dependence:

$$
\frac{\tilde{n}_{\mathrm{GAM}}}{n_{c}}=-\left(\sqrt{2} k_{r} \rho_{i} \frac{e \tilde{\Phi}}{T_{e}}\right) \sin (\theta) .
$$

Here $k_{r}$ is the radial mode number of the GAM, $\tilde{\Phi}$ the potential fluctuation, and $\rho_{i}$ the ion gyroradius. The density fluctuations at TEXTOR can be detected at $\theta=0^{\circ}$ and $\theta=90^{\circ}$ simultaneously. By reversing $B_{T}$ the direction of the $E \times B$ velocity is reversed, which yields the same conditions for the top antennas as in the case of $\theta=$ $270^{\circ}$ and unchanged magnetic field. For a qualitative analysis of the poloidal distribution, the amplitude of the peak at $f_{\text {GAM }}$ in the Fourier spectrum is estimated and shown in Fig. 4. A difference of the amplitude by a factor $\geq 10$ at midplane plane compared to the top position is observed at $\left(r_{c} / a=0.87\right)$. The nonvanishing amplitude at $\theta=0^{\circ}$ results from the poloidal resolution of the midplane antenna. In Fig. 4(b) the center frequency and the FWHM are shown. Reversing $B_{T}$ yields a pronounced GAM at $\theta=$ $270^{\circ}$, which is in qualitative agreement with Eq. (1).

For the quantitative estimation we use the approximation from [10]

$$
\frac{\langle\tilde{n}\rangle}{n_{c}} \approx\langle\tilde{\varphi}\rangle \frac{\lambda_{0}}{4 \pi \times 1.5 \times 2^{1 / 4} \sqrt{L_{n} \lambda_{r}}},
$$

where $\lambda_{0}$ is the probing wavelength, $\varphi$ the phase fluctua- 
\#98180 Top - midplane antennas comparison
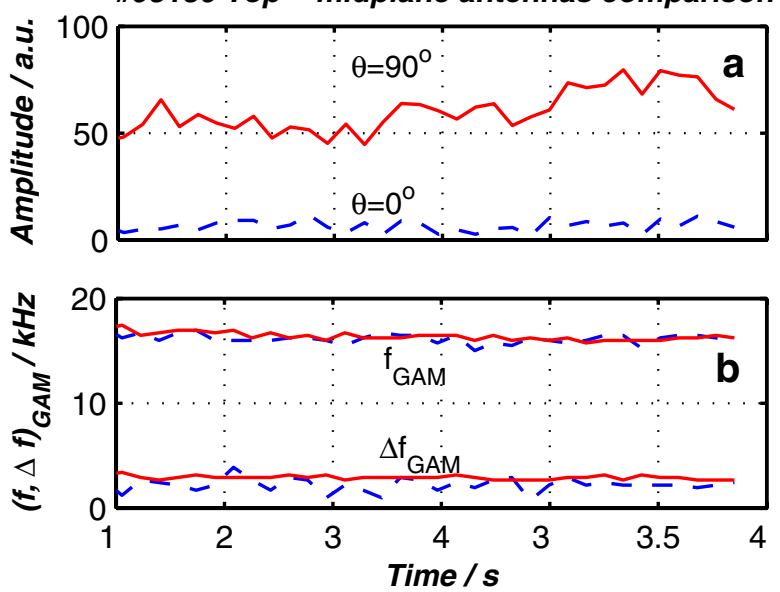

FIG. 4 (color online). (a) Comparison of the GAM amplitude at top (solid line) and midplane (dashed line) position. (b) Center frequency and FWHM of the GAM peak.

tions, $L_{n}$ the density scale length, and values in brackets denote the root mean square value. The radial wavelength $\left(\lambda_{r}\right)$ of the GAM is taken from the tokamak T-10 [9] measurements, which is justified since both machines are similar in geometrical dimensions as well as in the plasma parameters. For $r / a=0.87$ and $\lambda_{r} \approx 0.06 \mathrm{~m}$ we obtain $\left(\tilde{n} / n_{c}\right)_{\mathrm{GAM}} \approx 0.09 \% \pm 0.009 \%\left(\theta=90^{\circ}\right)$ and $\approx 0.008 \% \pm 0.008 \%\left(\theta=0^{\circ}\right)$, where the main error source results from the estimation of $\langle\tilde{\varphi}\rangle$. To estimate the total turbulence level in the range $5-500 \mathrm{kHz}$, the mean poloidal wavelength and the relationship $\lambda_{r} \approx 0.5 \lambda_{\theta}=$ $0.035 \mathrm{~m}[10,13]$ are used. We obtain $\left(\tilde{n} / n_{c}\right)_{\text {tot }} \approx 1.16 \%$ at $\theta=90^{\circ}$ and $1.55 \%$ at $\theta=0^{\circ}$, which yields $\tilde{n}_{\mathrm{GAM}} / \tilde{n}_{\mathrm{tot}} \approx 7.5 \%$ at $\theta=90^{\circ}$ and $\approx 0.5 \%$ at $\theta=0^{\circ}$.

The long-scale character of $\tilde{n}_{\mathrm{GAM}}$ oscillations implies the correlations between the equatorial and top antennas. Figure 5(a) shows the spectra for both signals; the coher-

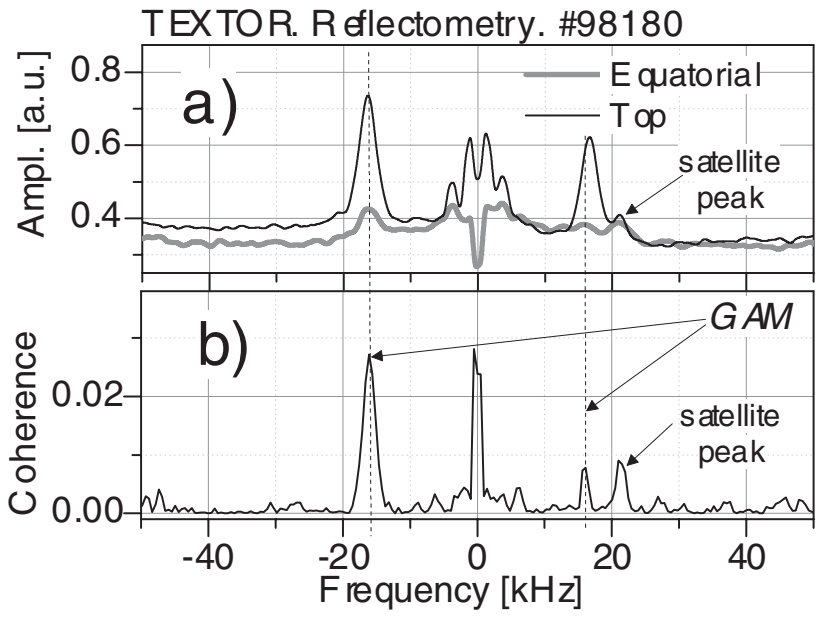

FIG. 5. Poloidal correlation for $\Delta \theta=90^{\circ}$. The GAM is visible in amplitude (a) and coherency (b) spectra. ence between them is shown in Fig. 5(b). All spectra exhibit pronounced peaks at $f_{\mathrm{GAM}} \approx \pm 17 \mathrm{kHz}$. The coherence level is several percent, but the contrast is high enough to claim that the same mode is observed at two poloidal angels simultaneously. The low coherence level is the consequence of the low amplitude of this mode at the midplane. Thus, the long-distance poloidal correlation validates the long-scale character of $\tilde{n}_{\mathrm{GAM}}$. Satellite peaks accompanying the GAM peak are often observed in the spectra. The peak at $f \approx 22 \mathrm{kHz}$ has no poloidal asymmetry [see Fig. 5(a)]. The corresponding peak in the coherence [Fig. 5(b)] indicates the same long-scale structure as the GAM. Similar observations are reported from T-10 $[6,9]$.

According to theory [2] the velocity of GAM flows changes periodically in absolute value and in sign. Owing to finite $\lambda_{r}$ these flows provide the additional shearing effect on the ambient turbulence, thus modulating its level $[5,6,8]$. The analysis of turbulence velocity oscillations $\left(\tilde{v}_{\mathrm{GAM}}\right)$ is done on the base of time-delay measurements for two pairs of the top antennas spaced poloidally at $\Delta \theta=0.025$ and $0.05 \mathrm{rad}$. In advance, the reflectometer signals are bandpass filtered $40 \leqq f \leqq 500 \mathrm{kHz}$, thus excluding any low frequency modes. The Fourier spectra for the time-delay series $\left[S(\Delta t)_{\Delta \theta=0.025}, S(\Delta t)_{\Delta \theta=0.050}\right]$ are shown in Fig. 6(a). While the period of GAM oscillations is $\approx 40-80 \mu \mathrm{s}$ and the acquisi>tion time is $1 \mu \mathrm{s}$, the correlation analysis is hampered by the short sample length $(\leq 15 \mu \mathrm{s})$ to resolve the period of the GAM. It results in a big scatter in the $\Delta t(t)$ series and high background in the spectra. The multiantenna system allows one to eliminate the uncorrelated background and to increase the contrast of the peak in the spectrum by use of coherence calculation [Fig. 6(b)]. The amplitude of $\tilde{v}_{\mathrm{GAM}}$ is evaluated from the peaks above the background in the spectra shown in Fig. 6(a). It amounts to $\sim 5 \%-10 \%$ of the ambient turbulence rotation. For the plasma under consideration the

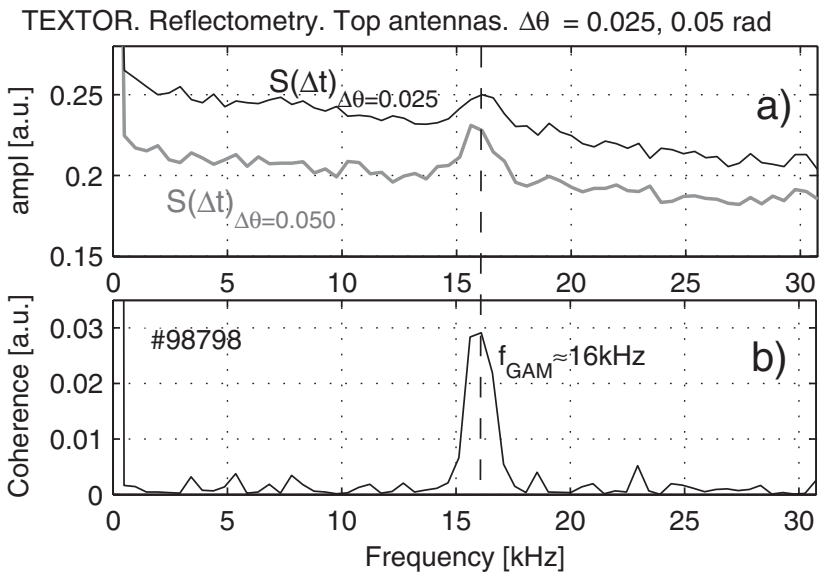

FIG. 6. The spectral analysis of the time-delay $\Delta t(t)$ series calculated for two pairs of the top antennas spaced poloidally at $\Delta \theta=0.025$ and 0.05 rad. (a) Fourier spectra; (b) coherence. 
TEXTOR. Reflectometry. \#98181, nfft=2048, $\mathrm{t}=[1.3 ; 2.3] \mathrm{s}$

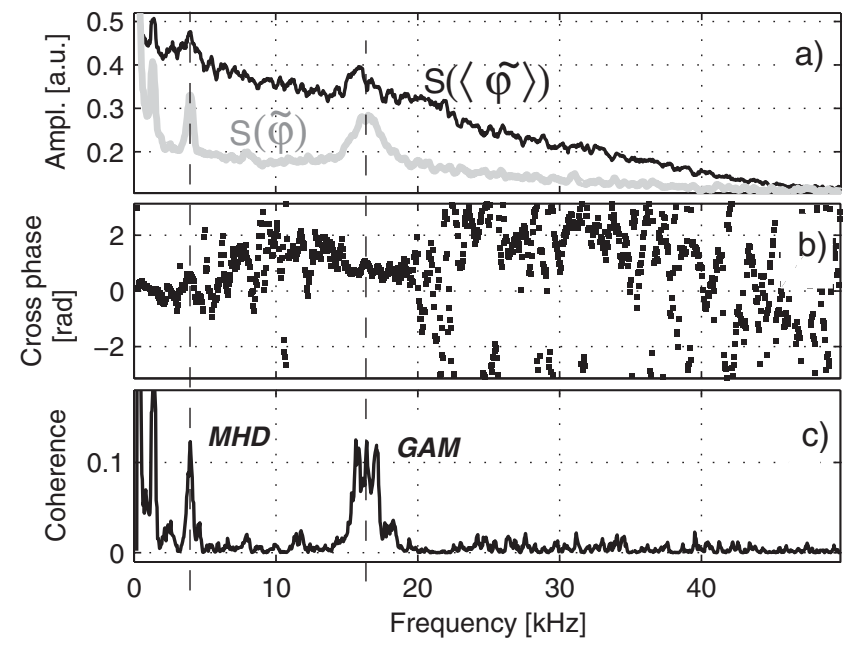

FIG. 7. The result of correlation analysis between the phase of reflected wave and its standard deviation: (a) amplitude spectra, (b) cross phase, and (c) coherence.

angular turbulence rotation $\left(\Omega \approx 1.2 \times 10^{4} \mathrm{rad} / \mathrm{s}\right)$ is approximately constant over the range of the cutoff positions $\left(r_{c}=0.3-0.4 \mathrm{~m}\right)$. As shown in Ref. [14] the turbulence rotation coincides with the plasma equilibrium rotation. The shearing rate is given by $\omega^{\prime}=\partial v / \partial r=\partial(\Omega r) / \partial r$. The instantaneous shearing rate in the presence of GAMs amounts to $\approx 2.2-4.8 \times 10^{4} \mathrm{~s}^{-1}$. Taking into account the GAM frequency, the effective shearing rate according to [15] is reduced by $\approx 5 \%$. The resulting shearing rate is less but comparable with the turbulence decorrelation rate of $\approx 8-10 \times 10^{4} \mathrm{~s}^{-1}$. Therefore we expect that the GAMs can regulate the ambient turbulence without totally suppressing it.

The influence of the GAM flows on the ambient turbulence level in frequency range $50 \leqq f \leqq 500 \mathrm{kHz}$ is investigated. The phase fluctuations $\tilde{\varphi}$ (which can be interpreted in terms of $\tilde{n}$ ) and its rms level $\langle\tilde{\varphi}\rangle$ are Fourier transformed. The amplitude of Fourier coefficients, cross phase, and coherence are shown in Figs. 7(a)-7(c), respectively. Note that (i) the peaks in the $S(\tilde{\varphi})$ and $S(\langle\tilde{\varphi}\rangle)$ spectra are at the same frequency of $\approx 17 \mathrm{kHz}$, and that (ii) the peak in the coherence spectrum at $f \approx 17 \mathrm{kHz}$ exceeds the background by $\sim 8-10$ times. It is a clear evidence for cause-and-effect relation between the GAM flows and the ambient turbulence level. The derivation of the cross phase in the range $15-18 \mathrm{kHz}$ is close to zero, which corresponds to zero time delay which is natural. The influence of the turbulence level by the MHD mode (see peak at $f_{\text {MHD }} \approx$ $4 \mathrm{kHz}$ ) is expected and was observed at TEXTOR earlier [10].

In summary, the coherent density oscillations, localized at the plasma edge, are observed reproducibly for the range of Ohmic plasma conditions. The frequency of the observed mode in $\tilde{n}$ obeys the theory predicted GAM scaling with local temperature and ion mass. The measurements of the GAM amplitude at several poloidal positions are in a good qualitative agreement with the theoretically predicted $\sin (\theta)$ (or $m=1$ ) distribution. The phase coherence over $\Delta \theta \approx 90^{\circ}$ confirms the long-scale nature of observed density oscillations, which is also consistent with the predicted $m=1$ structure. The oscillations of turbulence rotation $\tilde{v}_{\theta}$ related with the GAM are found. Its level is $\approx 5 \%-10 \%$ of the ambient turbulence rotation. The resulting shearing rate is comparable with the decorrelation rate of ambient turbulence. Indications are found that the $\tilde{v}_{\mathrm{GAM}}$ flows regulate the level of the ambient plasma turbulence.

The authors like to thank A. Melnikov and V. Vershkov for their interest and stimulating discussions.

[1] P. Diamond et al., Plasma Phys. Controlled Fusion 47, R35 (2005).

[2] N. Winsor et al., Phys. Fluids 11, 2448 (1968).

[3] A. Fujisawa et al., Phys. Rev. Lett. 93, 165002 (2004).

[4] Y. Hamada et al., Nucl. Fusion 45, 81 (2005).

[5] G. McKee et al., Plasma Phys. Controlled Fusion 45, A477 (2003).

[6] V. Vershkov et al., Nucl. Fusion 45, S203 (2005).

[7] G. McKee et al., Phys. Plasmas 10, 1712 (2003).

[8] G. Conway et al., Plasma Phys. Controlled Fusion 47, 1165 (2005).

[9] A. Melnikov et al., Plasma Phys. Controlled Fusion 48, S87 (2006).

[10] A. Kraemer-Flecken et al., Nucl. Fusion 44, 1143 (2004).

[11] T. S. Hahm et al., Plasma Phys. Controlled Fusion 42, A205 (2000).

[12] T. Ido et al., Plasma Phys. Controlled Fusion 48, S41 (2006).

[13] R. Fonck et al., Plasma Phys. Controlled Fusion 34, 1993 (1992).

[14] A. Kraemer-Flecken et al., Nucl. Fusion (to be published).

[15] T. S. Hahm et al., Phys. Plasmas 6, 922 (1999). 